1087 LSE OF A MICOCOMPUTER IN A DIAGNOSTIC VIROLOGY LABORATORY Robert P. Drucker, Catherine M. Wilfert Aseries of computer programs was developed using an existing data base management system (KnowledgeMan) on a microcomputer to assist with the management of a diagnostic virology lab. The primary purpose was to handle the daily routine: recording specimens and patient data, generating worksheets for the reading and processing of cultures, retrieving culture results or current status, and printing final report forms. It can also handle other lab procedures: Chlamydia cultures, Clostridium difficile toxin assays, and some serologic testing. In addition, the computer facilitates surveillance studies. It can rapidly generate a list of cultures or results for user-defined conditions: ages, hospital location, dates, or a variety of other parameters. A statistics package is included for simple analyses. Security of patient data is always a concern with computer use because of the ease of transporting large volumes of information on floppy disks. Security is provided by KnowledgeMan. A1l information is stored in scrambled form within files, and cannot be read if accessed from outside KnowledgeMan. On entering the system, each user is assigned a priority status. Each data type, age, result, is assigned up to 16 different access codes to restrict the user to certain pieces of information. The series of programs is menu-driven, and requires no computer knowledge by lab personnel. A computer can decrease significantly the paperwork done by hand, file large amounts of information in a sma11 space, facilitate epidemiologic surveys, and provide a rapid review and analysis of the work performed by the laboratory.

\section{$\dagger 1088$}

CLINICAI CORRELATIONS OF CSF ENDOTOXIN-LIKE ACTIVITY St. Louis university school of Medicine (spon. by Thomas Aceto). dren's Hospital, Department of Pediatrics/Adolescent Medicine, St. Louis, MO.

Detection of endotoxin-like activity (ELA) in cerebrospinal fluid (CSF) by limulus amebocyte lysate (LAL) cerebrospinal suggested to be a useful technique for diagnosis of gram-negative meningitis. We prospectively meningitis. We prospectively screened 1503 CSF specimens with a microassay utilizing $20 \mu \mathrm{l}$ of CSF added to $20 \mu \mathrm{l}$ of limulus reagent on an endotoxin-free slide. Specimens and controls were observed for gel formation after 60 minutes incubation at $37^{\circ} \mathrm{C}$ in a moisture chamber. Serial dilutions of CSF were used to quantitate ELA. The limit of sensitivity of the assay was $0.01 \mathrm{ng} / \mathrm{ml}$. All ELA positive ( $(t)$ specimens were subjected to confirmatory retesting, after which $49(3.3 \%)$ were ELA $(+)$. Comparison with the 38 available culture results revealed that 33 specimens (878) were culture $(+)$, that 3 of the 5 culture negative (-) specimens were from patients on therapy 5 culture negative $(-)$ specimens were from patients on therapy for gram-negative meningitis and a fourth was from a neonate. Overall specificity of the test was
$99.6 \%$ with a $(+)$ predictive value $99.6 \%$ with a $(+)$ predictive value of $86.8 \%$. There was one false predictive value of $99.9 \%$. with the occurrence of seizures (p<0.05), levels $>3000$ ng/mi with peripheral leukopenia and hypoglycorrhachia ( $0<0.02)$, and levels $>5 \times 10^{4} \mathrm{ng} / \mathrm{ml}$ with death $(p<0.005)$. We conclude that LAL micromethod gelation test is a sensitive, specific, simple

screening test for gram-negative meningitis that exhibits prog-
nostic value.

A SOLLUTION TO THE PERTUSSIS PROBLEM? Kathryn $M$.

$\bullet 1089$

Edwards, Eileen M. Lawrence, Peter F. Wright, Vanderbilt

The conventional Bordetel la pertussis vaccine consisting of killed whole. organisms has been shown to be effective in preventing disease but as sociated with significant local and systemic reactions pertussis vaccine containing two protein antigens, filamentous heelula tinin (F-HA) and leukocytosis promoting fact or hemagglutinin (LPF-HA) has been developed. The following study was designed to compare the immunogenicity and reactogenicity of the two vaccines. Thirty seven immunogenicity and reactogenicity of the two vaccines. Thirty seven children, ages $4-6$ years previously immunized with conventional DPT
vaccine at $2,4,6$ and 18 months, were enrolled just prior to administration vaccine at $2,4,6$ and 18 months, were enrolled just prior to administration of a pre-school DPT booster. Children were randomly assigned to receive either conventional pertussis or acellular pertussis vaccine in a double blind fashion. The dipht heria and tetanus components in both preparations were identical. Eighteen children received conventional pertussis while nineteen received acellular pertussis vaccine. Reaction rates were assessed by examinations by a single observer at 24 hours. Acellular pertussis vaccine was signi ficantly less reactogenic $(0<.05)$ for each of $:$ the signs and symptoms listed below.
Temp $>$ Fret- Abnormal Indura- Redness

\begin{tabular}{|c|c|c|c|c|c|c|}
\hline$\frac{V a c}{\text { con }}$ & $\begin{array}{l}\text { Temp> } \\
1.00 .4 \\
\text { al } 45 \%\end{array}$ & $\frac{\text { Pain }}{60 \%}$ & $\begin{array}{c}\begin{array}{c}\text { Fret- } \\
\text { fulness }\end{array} \\
45 \%\end{array}$ & $\begin{array}{c}\text { Abnormal } \\
\text { Gait } \\
\end{array}$ & $\begin{array}{c}\begin{array}{c}\text { Indura- } \\
\text { tion }>4 \mathrm{~cm}\end{array} \\
55 \%\end{array}$ & $\begin{array}{c}\text { Redness } \\
>4 \mathrm{~cm} \\
\end{array}$ \\
\hline & & $0 \%$ & $0 \%$ & $\begin{array}{r}5 \% \\
5 \%\end{array}$ & $\begin{array}{r}55 \% \\
5 \%\end{array}$ & \\
\hline
\end{tabular}
$\begin{array}{lcccccc}\text { acellular } & 10 \% & 0 \% & 0 \% & 5 \% & 5 \% & 0 \% \\ \text { Sera were obtained before and one month after vaccine administration. }\end{array}$ Equivalent rises in immunoagglutination titers and antibodies to F-HA and LPF-HA were measured in both groups. If studies in progressively younger children conf irm the reduced reactogenicity and the equal immunogonicity of the acellular vaccine, it may be a more appropriate candidate than the current vaccine.
1090 ANTIGENICALLY DISTINCT ROTAVIRUSES ARE COMMON CAUSES OF DIARRHEA IN HUMANS. Joseph Pediatrics and Comparative Medicine, Johns Hopkins University School of Medicine, Baltimore, MD 21205.

Rotaviruses (RV) are major causes of diarrheal disease in humans and other animals. Until recently, it was thought that all RVs shared a common antigen, and a vaccine based on this principle is undergoing field testing in humans. However, rotavirus-like agents (RVLA) which are morphologically indistinguishable from RVs but which do not share any antigenic similarity to standard RVs have recently been isolated from cows, piglets, birds, and humans. The role of these agents in
human disease has not been extensively studied.

We recently discovered a RVLA which is capable of infecting infant rats (J. Virol. 52:94-98, 1984). We found that fecal samples from adults and humans with acute gastroenteritis can transmit an identical disease to the infant rats. The infection is characterized by typical pathological changes in the gastrointestinal epithelial cells and by the passage of viral particles with a characteristic pattern of doublestranded, segmented RNA. We have devised enzyme immunoassays for the detection of RVLA antigen and antibody. The application of these assays to human specimens has indicated that RVLA infection is common in young children and that infection with RVLA can be associated with severe gastroenteritis. RVLA may be an important new agent of gastroenteritis in humans and animals.

\section{CYTOMEgaloviruS (CMV) ANTIGENIC} HETEROGENEITY MAY CAUSE FALSE NEGATIVE RESULTS IN ANTIBODY ASSAYS. Roger G. Faix.
RES Department of Pediatrics, University of Michigan Medical Center, Ann Arbor. (Spon. by SM Donn).

CMV antibody status may be an important factor in antenatal counselling and screening blood products and transplant donors. Though CMV strains are known to have heterogeneous antigens, most clinical laboratories use only a single antigen for CMV serology. The limitations of a single antigen in CMV serology were investigated.

Cord sera and antepartum maternal sera from 3 congenitally CMVinfected infants were CMV-seronegative (titer $<8$ ) on complement fixation (CF) assay using a commercial glycine-extracted (GE) AD169 antigen. When their own $\mathrm{CMV}$ isolates were propagated and made into GE-CF antigen, all became seropositive (cord titers $64,64,256$ maternal titers $16,32,32$ ). When 108 random cord sera were assayed for $\mathrm{CF}$ antibody using antigens AD169, Davis, and TC (a locally-derived antigen from one of the above infants), 44 were seropositive and 54 seronegative with all 3 antigens. Of the remaining 10 sera, 4 were positive with TC only, 3 with TC and Davis, 2 with Davis and 4 were and 1 with AD169 only. All 10 were positive when Davis and AD169, 3 antigens was used. No anticomplementary activity was detectable in 3 antigens was used. No anticomplementary activity was detectable in
any of these sera. A commercial CMV-indirect hemagglutination any of these sera. A commercial CMV-indirect hemagglutination
antibody assay that uses AD169 antigen was positive with only 4 of these 10 sera.

Antigenic heterogeneity among CMV strains may cause up to $7 \%$ of sera to be misclassified as seronegative if only a single antigen is used in the CF assay.

ANTIBODY RESPONSE TO PNEUMOCOCCAL VACCINE IN MAL102 NOURISHED CHILDREN. Senih M. Fikrig, Izzet Berkel and Microbiology, Downstate Medical Center and University of and Microbiology, Downstat

\section{cettepe, Ankara, Turkey.}

Twelve normal and sixteen malnourished (total calorie) children ( 6 with $<3 \%, 5$ with $<10 \%$ and 5 with $<25 \%$ malnourishment) over 2 years of age were vaccinated with pneumococcal vaccine containing 14 types of purified capsular polysaccharides. Pre and 2 to 3 weeks post-vaccination sera were obtained and assayed for antibody production against 12 capsular types by means of radioimmunoassay. Geometric mean antibody titers (GMT's) before immunization were comparable between the contro subjects and children with various degrees of malnourishment. Control subjects responded well to all capsular types (with the exception of type $6 \mathrm{~A}$ and $19 \mathrm{~F}$ known to be nonimmunogenic in the particular lot). Similarly malnourished children, irrespective of the degree of malnourishment showed a comparable level of antibody response to the same capsular polysaccharide types. Thus total calorie malnutrition - up to $25 \%$ - does not impair antibody production to pneumococcal polysaccharides and can be safely and effectively used in the protection of these 